International Journal of Pure and Applied Mathematics

Volume 102 No. 2 2015, 383-406

ISSN: 1311-8080 (printed version); ISSN: 1314-3395 (on-line version)

url: http://www.ijpam.eu

doi: http://dx.doi.org/10.12732/ijpam.v102i2.14

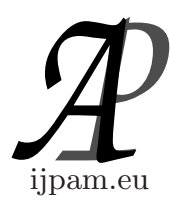

\title{
COMMON FIXED POINTS FOR COMPATIBLE MAPPINGS AND ITS VARIANTS IN MULTIPLICATIVE METRIC SPACES
}

\author{
Shin Min Kang ${ }^{1}$, Parveen Kumar $^{2}$, Sanjay Kumar ${ }^{3}$, \\ Poonam Nagpal ${ }^{4}$, Sudhir Kumar Garg ${ }^{5}$ \\ ${ }^{1}$ Department of Mathematics and RINS \\ Gyeongsang National University \\ Jinju, 660-701, KOREA \\ 2,3,4,5 Department of Mathematics \\ Deenbandhu Chhotu Ram University of Science and Technology \\ Murthal, Sonipat 131039, Haryana, INDIA
}

\begin{abstract}
In this paper, we introduce the notions of compatible mappings and its variants in multiplicative metric spaces. Next, we prove common fixed point theorems for these mappings.
\end{abstract}

AMS Subject Classification: 47H10, 54H25

Key Words: multiplicative metric space, compatible mapping, compatible mapping of type $(A)$, type $(B)$, type $(C)$ and type $(P)$

\section{Introduction and Preliminaries}

It is well know that the set of positive real numbers $\mathbb{R}^{+}$is not complete according to the usual metric. To overcome this problem, in 2008, Bashirov et al. [1] introduced the concept of multiplicative metric spaces as follows:

Received: April 25, 2015

(c) 2015 Academic Publications, Ltd.

${ }^{\S}$ Correspondence author url: www.acadpubl.eu 
Definition 1.1. Let $X$ be a nonempty set. A multiplicative metric is a mapping $d: X \times X \rightarrow \mathbb{R}^{+}$satisfying the following conditions:

(i) $d(x, y) \geq 1$ for all $x, y \in X$ and $d(x, y)=1$ if and only if $x=y$;

(ii) $d(x, y)=d(y, x)$ for all $x, y \in X$;

(iii) $d(x, y) \leq d(x, z) \cdot d(z, y)$ for all $x, y, z \in X$ (multiplicative triangle inequality).

Example 1.2. ([4]) Let $\mathbb{R}_{+}^{n}$ be the collection of all $n$-tuples of positive real numbers. Let $d: \mathbb{R}_{+}^{n} \times \mathbb{R}_{+}^{n} \rightarrow \mathbb{R}$ be defind as follows:

$$
d(x, y)=\left(\left|\frac{x_{1}}{y_{1}}\right| \cdot\left|\frac{x_{2}}{y_{2}}\right| \cdots\left|\frac{x_{n}}{y_{n}}\right|\right),
$$

where $x=\left(x_{1}, \ldots, x_{n}\right), y=\left(y_{1}, \ldots, y_{n}\right) \in \mathbb{R}_{+}^{n}$ and $|\cdot|: \mathbb{R}_{+} \rightarrow \mathbb{R}_{+}$is defined by

$$
|a|= \begin{cases}a & \text { if } a \geq 1 \\ \frac{1}{a} & \text { if } a<1\end{cases}
$$

Then it is obvious that all conditions of multiplicative metric are satisfied.

Example 1.3. ([5]) Let $d: \mathbb{R} \times \mathbb{R} \rightarrow[1, \infty)$ be defined as $d(x, y)=a^{|x-y|}$ for all $x, y \in X$, where $a>1$. Then $d$ is a multiplicative metric and hence $(X, d)$ is a multiplicative metric space.

One can refer to [2] and [4] for detailed the multiplicative metric topology.

Definition 1.4. Let $(X, d)$ be a multiplicative metric space. Then a sequence $\left\{x_{n}\right\}$ in $X$ said to be

(1) a multiplicative convergent to $x$ if for every multiplicative open ball $B_{\epsilon}(x)=\{y \mid d(x, y)<\epsilon\}, \epsilon>1$, there exists a natural number $N$ such that $n \geq N$, then $x_{n} \in B_{\epsilon}(x)$, that is, $d\left(x_{n}, x\right) \rightarrow 1$ as $n \rightarrow \infty$.

(2) a multiplicative Cauchy sequence if for all $\epsilon>1$, there exists $N \in \mathbb{N}$ such that $d\left(x_{n}, x_{m}\right)<\epsilon$ for all $m, n>N$, that is, $d\left(x_{n}, x_{m}\right) \rightarrow 1$ as $n \rightarrow \infty$.

(3) We call a multiplicative metric space complete if every multiplicative Cauchy sequence in it is multiplicative convergent to $x \in X$.

In 2012, Özavsar [4] gave the concept of multiplicative contraction mappings and proved some fixed point theorem of such mappings in a multiplicative metric space. 
Definition 1.5. Let $f$ be a mapping of a multiplicative metric space $(X, d)$ into itself. Then $f$ is called a multiplicative contraction if there exists a real constant $\lambda \in[0,1)$ such that

$$
d(f x, f y) \leq d^{\lambda}(x, y) \quad \text { for all } x, y \in X .
$$

Gu et. al. [3] introduced the notion of commuting and weakly commuting mappings in a multiplicative metric space and proved some fixed point theorems for these mappings.

Definition 1.6. Let $f$ and $g$ be two mappings of a multiplicative metric space $(X, d)$ into itself. Then $f$ and $g$ are called

(1) commuting mappings if $f g x=g f x$ for all $x \in X$.

(2) weakly commuting mappings if $d(f g x, g f x) \leq d(f x, g x)$ for all $x \in X$.

Remark 1.7. Notice that commuting mappings are obviously weakly commuting. However, the converse need not be true.

Example 1.8. Let $X=[0,1]$ be usual multiplicative metric $d$ on $X$. Define constant mappings $f$ and $g: X \rightarrow X$ by $f x=a$ and $g x=b$ for all $x \in X$, where $a, b \in[0,1]$ with $a \neq b$. Then $f$ and $g$ are weakly commuting but not commuting since

$$
d(f g x, g f x)=\left|\frac{a}{b}\right| \leq d(f x, g x)
$$

for all $x \in X$.

Example 1.9. Let $X=[0,1]$ be a multiplicative metric $d$ on $X$ defined by $d(x, y)=a^{|x-y|}$ for all $x, y \in X$, where $a>1$. Define mappings $f$ and $g: X \rightarrow X$ by $f x=\frac{x}{3-x}$ and $g x=\frac{x}{3}$ for all $x \in X$. For any $x \in X$,

$$
d(f g x, g f x)=a^{\left|\frac{2 x^{2}}{(9-x)(9-3 x)}\right|} \leq a^{\left|\frac{x^{2}}{9-3 x}\right|}=d(f x, g x) .
$$

Then $f$ and $g$ are weakly commuting but $f$ and $g$ are not commuting since

$$
f g x=\frac{x}{9-x}<\frac{x}{9-3 x}=g f x
$$

for any non-zero $x \in X$. 


\section{Relationships and Properties of Compatible Mappings and Its Variants}

Now we introduce the notions of compatible mappings and its variants in the setting of multiplicative metric spaces as follows:

Definition 2.1. Let $f$ and $g$ be two mappings of a multiplicative metric space $(X, d)$ into itself. Then $f$ and $g$ are called

(1) compatible if $\lim _{n \rightarrow \infty} d\left(f g x_{n}, g f x_{n}\right)=1$, whenever $\left\{x_{n}\right\}$ is a sequence in $X$ such that $\lim _{n \rightarrow \infty} f x_{n}=\lim _{n \rightarrow \infty} g x_{n}=t$ for some $t \in X$.

(2) compatible of type $(A)$ if

$$
\lim _{n \rightarrow \infty} d\left(f g x_{n}, g g x_{n}\right)=1 \text { and } \lim _{n \rightarrow \infty} d\left(g f x_{n}, f f x_{n}\right)=1,
$$

whenever $\left\{x_{n}\right\}$ is a sequence in $X$ such that $\lim _{n \rightarrow \infty} f x_{n}=\lim _{n \rightarrow \infty} g x_{n}=t$ for some $t \in X$.

(3) compatible of type $(B)$ if

$$
\lim _{n \rightarrow \infty} d\left(f g x_{n}, g g x_{n}\right) \leq\left[\lim _{n \rightarrow \infty} d\left(f g x_{n}, f t\right) \cdot \lim _{n \rightarrow \infty} d\left(f t, f f x_{n}\right)\right]^{1 / 2}
$$

and

$$
\lim _{n \rightarrow \infty} d\left(g f x_{n}, f f x_{n}\right) \leq\left[\lim _{n \rightarrow \infty} d\left(g f x_{n}, g t\right) \cdot \lim _{n \rightarrow \infty} d\left(g t, g g x_{n}\right)\right]^{1 / 2},
$$

whenever $\left\{x_{n}\right\}$ is a sequence in $X$ such that $\lim _{n \rightarrow \infty} f x_{n}=\lim _{n \rightarrow \infty} g x_{n}=t$ for some $t \in X$.

(4) compatible of type $(C)$ if

$$
\begin{aligned}
& \lim _{n \rightarrow \infty} d\left(f g x_{n}, g g x_{n}\right) \\
& \leq\left[\lim _{n \rightarrow \infty} d\left(f g x_{n}, f t\right) \cdot \lim _{n \rightarrow \infty} d\left(f t, f f x_{n}\right) \cdot \lim _{n \rightarrow \infty} d\left(f t, g g x_{n}\right)\right]^{1 / 3}
\end{aligned}
$$

and

$$
\begin{aligned}
& \lim _{n \rightarrow \infty} d\left(g f x_{n}, f f x_{n}\right) \\
& \leq\left[\lim _{n \rightarrow \infty} d\left(g f x_{n}, g t\right) \cdot \lim _{n \rightarrow \infty} d\left(g t, g g x_{n}\right) \cdot \lim _{n \rightarrow \infty} d\left(g t, f f x_{n}\right)\right]^{1 / 3},
\end{aligned}
$$

whenever $\left\{x_{n}\right\}$ is a sequence in $X$ such that $\lim _{n \rightarrow \infty} f x_{n}=\lim _{n \rightarrow \infty} g x_{n}=t$ for some $t \in X$.

(5) compatible of type $(P)$ if $\lim _{n \rightarrow \infty} d\left(f f x_{n}, g g x_{n}\right)=1$, whenever $\left\{x_{n}\right\}$ is a sequence in $X$ such that $\lim _{n \rightarrow \infty} f x_{n}=\lim _{n \rightarrow \infty} g x_{n}=t$ for some $t \in X$. 
Next we give relationships and properties of compatible mappings and its variants.

Proposition 2.2. Let $f$ and $g$ be compatible mappings of type $(A)$. If one of $f$ and $g$ is continuous, then $f$ and $g$ are compatible.

Proof. Since $f$ and $g$ be compatible of type $(A)$, so $1=\lim _{n \rightarrow \infty} d\left(f g x_{n}, g g x_{n}\right)$ and $1=\lim _{n \rightarrow \infty} d\left(g f x_{n}, f f x_{n}\right)$, whenever $\lim _{n \rightarrow \infty} f x_{n}=\lim _{n \rightarrow \infty} g x_{n}=t$ for some $t \in X$.

Suppose that $f$ is continuous. Then $\lim _{n \rightarrow \infty} f f=\lim _{n \rightarrow \infty} f g x_{n}=f t$ for some $t \in$ $X$. Now we get $\lim _{n \rightarrow \infty} d\left(f g x_{n}, g f x_{n}\right)=1$, i.e., $f$ and $g$ be compatible mappings.

Similarly, if $g$ is continuous, then $f$ and $g$ be compatible mappings.

Proposition 2.3. Every pair of compatible mappings of type $(A)$ is compatible of type $(B)$.

Proof. Suppose that $f$ and $g$ are compatible mappings of type $(A)$. Then we have

$$
\begin{aligned}
1 & =\lim _{n \rightarrow \infty} d\left(f g x_{n}, g g x_{n}\right) \\
& \leq\left[\lim _{n \rightarrow \infty} d\left(f g x_{n}, f t\right) \cdot \lim _{n \rightarrow \infty} d\left(f t, f f x_{n}\right)\right]^{1 / 2}
\end{aligned}
$$

and

$$
\begin{aligned}
1 & =\lim _{n \rightarrow \infty} d\left(g f x_{n}, f f x_{n}\right) \\
& \leq\left[\lim _{n \rightarrow \infty} d\left(g f x_{n}, g t\right) \cdot \lim _{n \rightarrow \infty} d\left(g t, g g x_{n}\right)\right]^{1 / 2},
\end{aligned}
$$

as derived.

Proposition 2.4. Let $f$ and $g$ be continuous mappings of a multiplicative metric space $(X, d)$ into itself. If $f$ and $g$ are compatible of type $(B)$, then they are compatible of type $(A)$.

Proof. Let $\left\{x_{n}\right\}$ be a sequence in $X$ such that $\lim _{n \rightarrow \infty} f x_{n}=\lim _{n \rightarrow \infty} g x_{n}=t$ for some $t \in X$. Since $f$ and $g$ are continuous, we have

$$
\begin{aligned}
\lim _{n \rightarrow \infty} d\left(f g x_{n}, g g x_{n}\right) & \leq\left[\lim _{n \rightarrow \infty} d\left(f g x_{n}, f t\right) \cdot \lim _{n \rightarrow \infty} d\left(f t, f f x_{n}\right)\right]^{1 / 2} \\
& =1
\end{aligned}
$$


and

$$
\begin{aligned}
\lim _{n \rightarrow \infty} d\left(g f x_{n}, f f x_{n}\right) & \leq\left[\lim _{n \rightarrow \infty} d\left(g f x_{n}, g t\right) \cdot \lim _{n \rightarrow \infty} d\left(g t, g g x_{n}\right)\right]^{1 / 2} \\
& =1 .
\end{aligned}
$$

Therefore, $f$ and $g$ compatible of type $(A)$. This completes the proof.

Proposition 2.5. Let $f$ and $g$ be continuous mappings of a multiplicative metric space $(X, d)$ into itself. If $f$ and $g$ are compatible of type $(B)$, then they are compatible.

Proof. Let $\left\{x_{n}\right\}$ be a sequence in $X$ such that $\lim _{n \rightarrow \infty} f x_{n}=\lim _{n \rightarrow \infty} g x_{n}=t$ for some $t \in X$. Since $f$ and $g$ are continuous, we have

$$
\lim _{n \rightarrow \infty} f f x_{n}=f t=\lim _{n \rightarrow \infty} f g x_{n}
$$

and

$$
\lim _{n \rightarrow \infty} g f x_{n}=g t=\lim _{n \rightarrow \infty} g g x_{n}
$$

By multiplicative triangle inequality, we have

$$
d\left(f g x_{n}, g f x_{n}\right) \leq d\left(f g x_{n}, g g x_{n}\right) \cdot d\left(g g x_{n}, g f x_{n}\right) .
$$

Letting $n \rightarrow \infty$ and taking into account that $f$ and $g$ are compatible of type $(B)$, we have

$$
\begin{aligned}
& \lim _{n \rightarrow \infty} d\left(f g x_{n}, g f x_{n}\right) \\
& \leq\left[\lim _{n \rightarrow \infty} d\left(f g x_{n}, f t\right) \cdot \lim _{n \rightarrow \infty} d\left(f t, f f x_{n}\right)\right]^{1 / 2} \cdot \lim _{n \rightarrow \infty} d\left(g g x_{n}, g f x_{n}\right) \\
& =1 .
\end{aligned}
$$

Therefore, $f$ and $g$ are compatible. This completes the proof.

Proposition 2.6. Let $f$ and $g$ be continuous mappings of a multiplicative metric space $(X, d)$ into itself. If $f$ and $g$ are compatible, then they are compatible of type $(B)$.

Proof. Since $f$ and $g$ are compatible so there exists $\left\{x_{n}\right\}$ be a sequence in $X$ such that $\lim _{n \rightarrow \infty} f x_{n}=\lim _{n \rightarrow \infty} g x_{n}=t$ for some $t \in X$ for which $\lim _{n \rightarrow \infty} d\left(f g x_{n}, g f x_{n}\right)=$ 1. Since $f$ and $g$ are continuous, we have

$$
\lim _{n \rightarrow \infty} f f x_{n}=f t=\lim _{n \rightarrow \infty} f g x_{n}
$$


and

$$
\lim _{n \rightarrow \infty} g f x_{n}=g t=\lim _{n \rightarrow \infty} g g x_{n},
$$

so

$$
\lim _{n \rightarrow \infty} f f x_{n}=\lim _{n \rightarrow \infty} f g x_{n}=\lim _{n \rightarrow \infty} g f x_{n}=\lim _{n \rightarrow \infty} g g x_{n} .
$$

Now

$$
\lim _{n \rightarrow \infty} d\left(f g x_{n}, g g x_{n}\right) \leq\left[\lim _{n \rightarrow \infty} d\left(f g x_{n}, f t\right) \cdot \lim _{n \rightarrow \infty} d\left(f t, f f x_{n}\right)\right]^{1 / 2}
$$

and

$$
\lim _{n \rightarrow \infty} d\left(g f x_{n}, f f x_{n}\right) \leq\left[\lim _{n \rightarrow \infty} d\left(g f x_{n}, g t\right) \cdot \lim _{n \rightarrow \infty} d\left(g t, g g x_{n}\right)\right]^{1 / 2},
$$

which implies that $f$ and $g$ be compatible of type $(B)$.

Proposition 2.7. Let $f$ and $g$ be continuous mappings of a multiplicative metric space $(X, d)$ into itself. Then

(1) $f$ and $g$ are compatible if and only if they are compatible of type $(B)$;

(2) $f$ and $g$ are compatible of type $(A)$ if and only if they are compatible of type $(B)$.

Proof. (1) One can easily prove it using Propositions 2.5 and 2.6.

(2) One can easily prove it using Propositions 2.3 and 2.4.

Proposition 2.8. Let $f$ and $g$ be compatible mappings of a multiplicative metric space $(X, d)$ into itself. If $f t=g t$ for some $t \in X$, then $f g t=f f t=$ $g g t=g f t$.

Proof. Suppose that $\left\{x_{n}\right\}$ is a sequence in $X$ defined by $x_{n}=t, n=1,2, \ldots$ for some $t \in X$ and $f t=g t$. Then we have $f x_{n}, g x_{n} \rightarrow f t$ as $n \rightarrow \infty$. Since $f$ and $g$ are compatible, we have

$$
d(f g t, g f t)=\lim _{n \rightarrow \infty} d\left(f g x_{n}, g f x_{n}\right)=1 .
$$

Hence we have $f g t=g g t$. Therefore, since $f t=g t$, we have $f g t=f f t=g g t=$ $g f t$. This completes the proof.

From Proposition 2.8 we have 
Proposition 2.9. Let $f$ and $g$ be compatible mappings of a multiplicative metric space $(X, d)$ into itself. Suppose that $\lim _{n \rightarrow \infty} f x_{n}=\lim _{n \rightarrow \infty} g x_{n}=t$ for some $t \in X$. Then

(a) $\lim _{n \rightarrow \infty} g f x_{n}=f t$ if $f$ is continuous at $t$.

(b) $\lim _{n \rightarrow \infty} f g x_{n}=g t$ if $g$ is continuous at $t$.

(c) $f g t=g f t$ and $f t=g t$ if $f$ and $g$ are continuous at $t$.

Proof. (a) Suppose that $f$ is continuous at $t$. Since $\lim _{n \rightarrow \infty} f x_{n}=\lim _{n \rightarrow \infty} g x_{n}=t$ for some $t \in X$, we have $f g x_{n} \rightarrow f t$ as $n \rightarrow \infty$. Since $f$ and $g$ are compatible, we have

$$
\begin{aligned}
\lim _{n \rightarrow \infty} d\left(g f x_{n}, f t\right) & =\lim _{n \rightarrow \infty} d\left(g f x_{n}, f g x_{n}\right) \cdot \lim _{n \rightarrow \infty} d\left(f g x_{n}, f t\right) \\
& =1 .
\end{aligned}
$$

Therefore, $\lim _{n \rightarrow \infty} g f x_{n}=f t$. This completes the proof.

(b) The proof of $\lim _{n \rightarrow \infty} f g x_{n}=g t$ follows by similar arguments as in $(a)$.

(c) Suppose that $f$ and $g$ are continuous at $t$. Since $g x_{n} \rightarrow t$ as $n \rightarrow \infty$ and $f$ is continuous at $t$, by $(a), g f x_{n} \rightarrow f t$ as $n \rightarrow \infty$. On the other hand, $g$ is also continuous at $t, g f x_{n} \rightarrow g t$. Thus, we have $f t=g t$ by the uniqueness of limit and so by Proposition 2.8, $f g t=g f t$. This completes the proof.

Proposition 2.10. Let $f$ and $g$ be compatible mappings of type $(B)$ of a multiplicative metric space $(X, d)$ into itself. If $f t=g t$ for some $t \in X$, then $f g t=f f t=g g t=g f t$.

Proof. Suppose that $\left\{x_{n}\right\}$ is a sequence in $X$ defined by $x_{n}=t, n=1,2, \ldots$ for some $t \in X$ and $f t=g t$. Then we have $f x_{n}, g x_{n} \rightarrow f t$ as $n \rightarrow \infty$. Since $f$ and $g$ are compatible of type $(B)$, we have

$$
\begin{aligned}
d(f g t, g g t) & =\lim _{n \rightarrow \infty} d\left(f g x_{n}, g g x_{n}\right) \\
& \leq\left[\lim _{n \rightarrow \infty} d\left(f g x_{n}, f f t\right) \cdot \lim _{n \rightarrow \infty} d\left(f f t, f f x_{n}\right)\right]^{1 / 2} \\
& =1 .
\end{aligned}
$$

Hence we have $f g t=g g t$. Therefore, since $f t=g t$, we have $f g t=f f t=g g t=$ gft. This completes the proof.

From Proposition 2.10 we have 
Proposition 2.11. Let $f$ and $g$ be compatible mappings of type $(B)$ of a multiplicative metric space $(X, d)$ into itself. Suppose that $\lim _{n \rightarrow \infty} f x_{n}=$ $\lim _{n \rightarrow \infty} g x_{n}=t$ for some $t \in X$. Then

(a) $\lim _{n \rightarrow \infty} g g x_{n}=f t$ if $f$ is continuous at $t$.

(b) $\lim _{n \rightarrow \infty} f f x_{n}=g t$ if $g$ is continuous at $t$.

(c) $f g t=g f t$ and $f t=g t$ if $f$ and $g$ are continuous at $t$.

Proof. (a) Suppose that $f$ is continuous at $t$. Since $\lim _{n \rightarrow \infty} f x_{n}=\lim _{n \rightarrow \infty} g x_{n}=t$ for some $t \in X$, we have $f f x_{n}, f g x_{n} \rightarrow f t$ as $n \rightarrow \infty$. Since $f$ and $g$ are compatible of type $(B)$, we have

$$
\begin{aligned}
\lim _{n \rightarrow \infty} d\left(f t, g g x_{n}\right) & =\lim _{n \rightarrow \infty} d\left(f g x_{n}, g g x_{n}\right) \\
& \leq\left[\lim _{n \rightarrow \infty} d\left(f g x_{n}, f t\right) \cdot \lim _{n \rightarrow \infty} d\left(f t, f f x_{n}\right)\right]^{1 / 2} \\
& =d(f t, f t)=1 .
\end{aligned}
$$

Therefore, $\lim _{n \rightarrow \infty} g g x_{n}=f t$. This completes the proof.

(b) The proof of $\lim _{n \rightarrow \infty} f f x_{n}=g t$ follows by similar arguments as in $(a)$.

(c) Suppose that $f$ and $g$ are continuous at $t$. Since $g x_{n} \rightarrow t$ as $n \rightarrow \infty$ and $f$ is continuous at $t$, by $(a), g g x_{n} \rightarrow f t$ as $n \rightarrow \infty$. On the other hand, $g$ is also continuous at $t, g g x_{n} \rightarrow g t$. Thus, we have $f t=g t$ by the uniqueness of limit and so by Proposition 2.10, fgt $=g f t$. This completes the proof.

Remark 2.12. In Proposition 2.10, assume that $f$ and $g$ be compatible mappings of type $(C)$ or of type $(P)$ instead of of type $(B)$. the conclusion of Proposition 2.10 still holds.

Remark 2.13. In Proposition 2.11, assume that $f$ and $g$ be compatible mappings of type $(C)$ or of type $(P)$ instead of of type $(B)$. the conclusion of Proposition 2.11 still holds.

Remark 2.14. Every weakly commuting mapping is compatible but converse need not be true.

Indeed, since $f$ and $g$ be weakly commuting mappings, therefore, $d(f g x, g f x) \leq$ $d(f x, g x)$ for all $x \in X$. Let $\lim _{n \rightarrow \infty} f x_{n}=\lim _{n \rightarrow \infty} g x_{n}=t$ for some $t \in X$. Then 
$d\left(f g x_{n}, g f x_{n}\right) \leq 1$, which implies that $\lim _{n \rightarrow \infty} d\left(f g x_{n}, g f x_{n}\right)=1$, i.e., $f$ and $g$ be compatible mappings.

Example 2.15. Consider $X=[0, \infty)$ with multiplicative metric $d(x, y)=$ $e^{|x-y|}$ on $X$. Define mappings $f$ and $g: X \rightarrow X$ by $f x=x^{3}$ and $g x=2 x^{3}$. Then $f$ and $g$ are compatible but not weakly commuting.

Remark 2.16. Notions of compatible mappings and its variants are independent to each other as follows.

Example 2.17. Let $X=\mathbb{R}$, the set of all real numbers, with the usual multiplicative metric $d$, define $f$ and $g: X \rightarrow X$ by

$$
f x=\left\{\begin{array}{ll}
\frac{1}{x^{4}} & \text { if } x \neq 0, \\
1 & \text { if } x=0
\end{array} \text { and } \quad g x= \begin{cases}\frac{1}{x^{2}} & \text { if } x \neq 0 \\
1 & \text { if } x=0 .\end{cases}\right.
$$

Then $f$ and $g$ are not continuous at $t=0$. Consider a sequence $\left\{x_{n}\right\}$ in $X$ defined by $x_{n}=n, n=1,2, \ldots$ Then for $n \rightarrow \infty$ we have $f x_{n}=\frac{1}{n^{4}} \rightarrow t=$ $0, \quad g x_{n}=\frac{1}{n^{2}} \rightarrow t=0$ and

$$
\lim _{n \rightarrow \infty} d\left(f g x_{n}, g f x_{n}\right)=\lim _{n \rightarrow \infty}\left|\frac{n^{8}}{n^{8}}\right|=1 .
$$

However, the following limits do not exist:

$$
\begin{aligned}
& \lim _{n \rightarrow \infty} d\left(f g x_{n}, g g x_{n}\right)=\lim _{n \rightarrow \infty}\left|\frac{n^{8}}{n^{4}}\right|=\infty, \\
& {\left[\lim _{n \rightarrow \infty} d\left(f g x_{n}, f 0\right) \cdot \lim _{n \rightarrow \infty} d\left(f 0, f f x_{n}\right)\right]^{1 / 2}} \\
& =\left[\lim _{n \rightarrow \infty}\left|\frac{n^{8}}{1}\right| \cdot \lim _{n \rightarrow \infty}\left|\frac{n^{16}}{1}\right|\right]^{1 / 2} \\
& =\infty
\end{aligned}
$$

and

$$
\begin{aligned}
& \lim _{n \rightarrow \infty} d\left(g f x_{n}, f f x_{n}\right)=\lim _{n \rightarrow \infty}\left|\frac{n^{16}}{n^{8}}\right|=\infty, \\
& {\left[\lim _{n \rightarrow \infty} d\left(g f x_{n}, g 0\right) \cdot \lim _{n \rightarrow \infty} d\left(g 0, g g x_{n}\right)\right]^{1 / 2}} \\
& =\left[\lim _{n \rightarrow \infty}\left|\frac{n^{8}}{2}\right| \cdot \lim _{n \rightarrow \infty}\left|\frac{n^{4}}{2}\right|\right]^{1 / 2} \\
& =\infty .
\end{aligned}
$$


Also $\lim _{n \rightarrow \infty} d\left(f g x_{n}, g g x_{n}\right)=\infty$ and $\lim _{n \rightarrow \infty} d\left(g f x_{n}, f f x_{n}\right)=\infty$ and we get

$$
\begin{aligned}
& {\left[\lim _{n \rightarrow \infty} d\left(f g x_{n}, f 0\right) \cdot \lim _{n \rightarrow \infty} d\left(f 0, f f x_{n}\right) \cdot \lim _{n \rightarrow \infty} d\left(f 0, g g x_{n}\right)\right]^{1 / 3}} \\
& =\left[\lim _{n \rightarrow \infty}\left|\frac{n^{8}}{1}\right| \cdot \lim _{n \rightarrow \infty}\left|\frac{n^{16}}{1}\right| \cdot \lim _{n \rightarrow \infty}\left|\frac{n^{4}}{1}\right|\right]^{1 / 3} \\
& =\infty
\end{aligned}
$$

and

$$
\begin{aligned}
& {\left[\lim _{n \rightarrow \infty} d\left(g f x_{n}, g 0\right) \cdot \lim _{n \rightarrow \infty} d\left(g 0, g g x_{n}\right) \cdot \lim _{n \rightarrow \infty} d\left(g 0, f f x_{n}\right)\right]^{1 / 3}} \\
& =\left[\lim _{n \rightarrow \infty}\left|\frac{n^{8}}{2}\right| \cdot \lim _{n \rightarrow \infty}\left|\frac{n^{4}}{2}\right| \cdot \lim _{n \rightarrow \infty}\left|\frac{n^{16}}{2}\right|\right]^{1 / 3} \\
& =\infty
\end{aligned}
$$

Also

$$
\lim _{n \rightarrow \infty} d\left(f f x_{n}, g g x_{n}\right)=\left|\frac{n^{12}}{1}\right|=\infty .
$$

Therefore, $f$ and $g$ are compatible but they are not compatible of type $(A)$, compatible of type $(B)$, type $(C)$ and of type $(P)$.

Example 2.18. Let $X=[0,6]$ with the usual multiplicative metric $d$, define $f$ and $g: X \rightarrow X$ by

$$
f x=\left\{\begin{array}{ll}
x & \text { if } x \in[0,3), \\
6 & \text { if } x \in[3,6]
\end{array} \text { and } g x= \begin{cases}6-x & \text { if } x \in[0,3), \\
6 & \text { if } x \in[3,6]\end{cases}\right.
$$

Then $f$ and $g$ are not continuous at $t=3$. Now, we assert that $f$ and $g$ are not compatible but they are compatible of type $(A)$, of type $(B)$, of type $(C)$ and of type $(P)$. To see this, suppose that $\left\{x_{n}\right\} \subset[0,6]$ and that $f x_{n}, g x_{n} \rightarrow t$. By definition of $f$ and $g, t \in[3,6]$. Since $f$ and $g$ agree on $[3,6]$, we have only to consider $t=3$. So we can suppose that $x_{n} \rightarrow 3$ and that $x_{n}<3$ for all $n$. Then $g x_{n}=6-x_{n} \rightarrow 3$ from the right and $f x_{n}=x_{n} \rightarrow 3$ from the left. Thus, since $x_{n}<3$ and $6-x_{n}>3$ for all $n$,

$$
\lim _{n \rightarrow \infty} d\left(f g x_{n}, g f x_{n}\right)=\left|\frac{6}{6-x_{n}}\right| \rightarrow 2 .
$$

Further, we have

$$
\lim _{n \rightarrow \infty} d\left(f g x_{n}, g g x_{n}\right)=\left|\frac{6}{6}\right|=1,
$$




$$
\begin{aligned}
& {\left[\lim _{n \rightarrow \infty} d\left(f g x_{n}, f 3\right) \cdot \lim _{n \rightarrow \infty} d\left(f 3, f f x_{n}\right)\right]^{1 / 2}} \\
& =\left[\lim _{n \rightarrow \infty}\left|\frac{6}{6}\right| \cdot \lim _{n \rightarrow \infty}\left|\frac{6}{x_{n}}\right|\right]^{1 / 2} \\
& =\sqrt{2}
\end{aligned}
$$

and

$$
\begin{aligned}
& \lim _{n \rightarrow \infty} d\left(g f x_{n}, f f x_{n}\right)=\left|\frac{6-x_{n}}{x_{n}}\right|=1, \\
& {\left[\lim _{n \rightarrow \infty} d\left(g f x_{n}, g 3\right) \cdot \lim _{n \rightarrow \infty} d\left(g 3, g g x_{n}\right)\right]^{1 / 2}} \\
& =\left[\lim _{n \rightarrow \infty}\left|\frac{6}{6-x_{n}}\right| \cdot \lim _{n \rightarrow \infty}\left|\frac{6}{6}\right|\right]^{1 / 2} \\
& =\sqrt{2} .
\end{aligned}
$$

Also $\lim _{n \rightarrow \infty} d\left(f g x_{n}, g g x_{n}\right)=1$ and $\lim _{n \rightarrow \infty} d\left(g f x_{n}, f f x_{n}\right)=1$ and we get

$$
\left[\lim _{n \rightarrow \infty} d\left(f g x_{n}, f 3\right) \cdot \lim _{n \rightarrow \infty} d\left(f 3, f f x_{n}\right) \cdot \lim _{n \rightarrow \infty} d\left(f 3, g g x_{n}\right)\right]^{1 / 3}=\sqrt[3]{2}
$$

and

$$
\left[\lim _{n \rightarrow \infty} d\left(g f x_{n}, g 3\right) \cdot \lim _{n \rightarrow \infty} d\left(g 3, g g x_{n}\right) \cdot \lim _{n \rightarrow \infty} d\left(g 3, f f x_{n}\right)\right]^{1 / 3}=\sqrt[3]{2}
$$

as $x_{n} \rightarrow 3$ and $\lim _{n \rightarrow \infty} f x_{n}=\lim _{n \rightarrow \infty} g x_{n}=3$. Also we have

$$
\lim _{n \rightarrow \infty} d\left(f f x_{n}, g g x_{n}\right)=\left|\frac{6}{6}\right|=1 .
$$

Therefore, $f$ and $g$ are compatible mappings of type $(A)$, of type $(B)$, of type $(C)$ and of type $(P)$ but they are not compatible.

\section{Fixed Points for Compatible Mappings and Its Variants}

In 2014, He et. al. [2] proved the common fixed points for pair of weak commutative mappings on a complete multiplicative metric spaces as follow: 
Theorem 3.1. Let $S, T, A$ and $B$ be mappings of a complete multiplicative metric space $(X, d)$ into itself satisfying the following conditions:

(C1) $S X \subset B X, \quad T X \subset A X$;

$$
\begin{aligned}
d(S x, T y) \leq & {[\max \{d(A x, B y), d(A x, S x),} \\
& d(B y, T y), d(S x, B y), d(A x, T y)\}]^{\lambda}
\end{aligned}
$$

for all $x, y \in X$, where $\lambda \in\left(0, \frac{1}{2}\right)$.

(C3) one of the mappings $S, T, A$ and $B$ is continuous.

Assume that the pairs $(A, S)$ and $(B, T)$ are weakly commuting. Then $S, T, A$ and $B$ have a unique common fixed point.

Now we give the following theorem for compatible mappings.

Theorem 3.2. Let $S, T, A$ and $B$ be mappings of a complete multiplicative metric space $(X, d)$ into itself satisfying $(C 1)-(C 3)$.

Assume that the pairs $(A, S)$ and $(B, T)$ are compatible. Then $S, T, A$ and $B$ have a unique common fixed point.

Proof. Since $S X \subset B X$. Now consider a point $x_{0} \in X$, there exists $x_{1} \in X$ such that $S x_{0}=B x_{1}=y_{0}$ for this point $x_{1}$ there exists $x_{2} \in X$ such that $T x_{1}=A x_{2}=y_{1}$. Continuing in this way, one can construct sequences such that

$$
y_{2 n}=S x_{2 n}=B x_{2 n+1}, \quad y_{2 n+1}=T x_{2 n+1}=A x_{2 n+2} .
$$

From the proof of [2, Theorem 3.1], $\left\{y_{n}\right\}$ is multiplicative Cauchy sequence in $X$. Consequently, the subsequences $\left\{S x_{2 n}\right\},\left\{A x_{2 n}\right\},\left\{T x_{2 n+1}\right\}$ and $\left\{B x_{2 n+1}\right\}$ of the sequence $\left\{y_{n}\right\}$ also converge to $z$.

Now suppose that $A$ is continuous. Then $A A x_{2 n}, A S x_{2 n}$ converge to $A z$ as $n \rightarrow \infty$. Since $(A, S)$ are compatible on $X$, it follows from Proposition 2.9 $S A x_{2 n}$ converges to $A z$ as $n \rightarrow \infty$.

We claim that $z=A z$. Consider

$$
\begin{aligned}
d\left(S A x_{2 n}, T x_{2 n+1}\right) \leq[ & \max \left\{d\left(A A x_{2 n}, B x_{2 n+1}\right), d\left(A A x_{2 n}, S A x_{2 n}\right),\right. \\
& d\left(B x_{2 n+1}, T x_{2 n+1}\right), d\left(S A x_{2 n}, B x_{2 n+1}\right), \\
& \left.\left.d\left(A A x_{2 n}, T x_{2 n+1}\right)\right\}\right]^{\lambda} .
\end{aligned}
$$

Letting $n \rightarrow \infty$, we have

$$
\begin{aligned}
d(A z, z) & \leq[\max \{d(A z, z), d(A z, A z), d(z, z), d(A z, z), d(A z, z)\}]^{\lambda} \\
& =d^{\lambda}(A z, z) .
\end{aligned}
$$


This implies that $d(A z, z)=1$ implies $A z=z$.

Next we claim that $S z=z$. Consider

$$
\begin{aligned}
d\left(S z, T x_{2 n+1}\right) \leq[ & \max \left\{d\left(A z, B x_{2 n+1}\right), d(A z, S z),\right. \\
& d\left(B x_{2 n+1}, T x_{2 n+1}\right), d\left(S z, B x_{2 n+1}\right), \\
& \left.\left.d\left(A z, T x_{2 n+1}\right)\right\}\right]^{\lambda} .
\end{aligned}
$$

Letting $n \rightarrow \infty$, we have

$$
\begin{aligned}
d(S z, z) & \leq[\max \{d(z, z), d(z, S z), d(z, z), d(S z, z), d(S z, z)\}]^{\lambda} \\
& =d^{\lambda}(S z, z) .
\end{aligned}
$$

This implies that $S z=z$. Since $S X \subset B X$ and hence there exists a point $u \in X$ such that $z=S z=B u$.

We claim that $z=T u$.

$$
\begin{aligned}
d(z, T u)= & d(S z, T u) \\
\leq & {[\max \{d(A z, B u), d(A z, S z),} \\
& \quad d(B u, T u), d(S z, B u), d(A z, T u)\}]^{\lambda} \\
= & {[\max \{d(z, z), d(z, z), d(z, T u), d(z, z), d(z, T u)\}]^{\lambda} . }
\end{aligned}
$$

This implies that $z=T u$. Since $(B, T)$ is compatible in $X$ and $B u=T u=z$, by Proposition 2.8, we have $B T u=T B u$ and hence $B z=B T u=T B u=T z$. Also, we have

$$
\begin{aligned}
d(z, B z)= & d(S z, T z) \\
\leq & {[\max \{d(A z, B z), d(A z, S z)} \\
& \quad d(B z, T z), d(S z, B z), d(A z, T z)\}]^{\lambda} \\
= & {[\max \{d(z, B z), d(z, z), d(B z, T z), d(z, B z), d(z, B z)\}]^{\lambda} . }
\end{aligned}
$$

This implies that $z=B z$. Hence, $z=B z=T z=A z=S z$. Therefore, $z$ is common fixed point of $S, T, A$ and $B$.

Similarly, we can also complete the proof when $B$ is continuous.

Next suppose that $S$ is continuous. Then $S S x_{2 n}, S A x_{2 n}$ converge to $A z$ as $n \rightarrow \infty$. Since $A$ and $S$ are compatible on $X$, it follows from Proposition 2.9 that $A S x_{2 n}$ converges to $A z$ as $n \rightarrow \infty$.

Consider

$$
\begin{aligned}
d\left(S S x_{2 n}, T x_{2 n+1}\right) \leq[ & \max \left\{d\left(A S x_{2 n}, B x_{2 n+1}\right), d\left(A S x_{2 n}, S S x_{2 n}\right),\right. \\
& d\left(B x_{2 n+1}, T x_{2 n+1}\right), d\left(S S x_{2 n}, B x_{2 n+1}\right) \\
& \left.\left.d\left(A S x_{2 n}, T x_{2 n+1}\right)\right\}\right]^{\lambda}
\end{aligned}
$$


Letting $n \rightarrow \infty$, we get

$$
\begin{aligned}
d(S z, z) & \leq[\max \{d(S z, z), d(S z, S z), d(z, z), d(S z, z), d(S z, z)\}]^{\lambda} \\
& =d^{\lambda}(S z, z),
\end{aligned}
$$

which implies that $S z=z$. Since $S X \subset B X$ and hence there exists a point $v \in X$ such that $z=S z=B w$. Consider

$$
\begin{aligned}
d\left(S S x_{2 n}, T v\right) \leq[ & \max \left\{d\left(A S x_{2 n}, B v\right), d\left(A S x_{2 n}, S S x_{2 n}\right),\right. \\
& \left.\left.d(B v, T v), d\left(S S x_{2 n}, B v\right), d\left(A S x_{2 n}, T v\right)\right\}\right]^{\lambda} .
\end{aligned}
$$

Letting $n \rightarrow \infty$, we have

$$
\begin{aligned}
d(z, T v) & \leq[\max \{d(z, z), d(z, z), d(z, T v), d(z, z), d(z, T v)\}]^{\lambda} \\
& =d^{\lambda}(z, T v) .
\end{aligned}
$$

This implies that $z=T v$. Since $B$ and $T$ are compatible on $X$ and $B v=T v=z$, by Proposition 2.8, we have $B T v=T B v$ and hence $B z=B T v=T B v=T z$. Consider

$$
\begin{aligned}
d\left(S x_{2 n}, T z\right) \leq & {\left[\operatorname { m a x } \left\{d\left(A x_{2 n}, B z\right), d\left(A x_{2 n}, S x_{2 n}\right),\right.\right.} \\
& \left.\left.d(B z, T z), d\left(S x_{2 n}, B z\right), d\left(A x_{2 n}, T z\right)\right\}\right]^{\lambda} .
\end{aligned}
$$

Letting $n \rightarrow \infty$, we get

$$
\begin{aligned}
d(z, T z) & \leq[\max \{d(z, T z), d(z, z), d(T z, T z), d(z, T z), d(z, T z)\}]^{\lambda} \\
& =d^{\lambda}(z, T z) .
\end{aligned}
$$

This implies that $T z=z$. Since $T X \subset A X$, so there exists a point $w \in X$ such that $z=T z=A w$. Consider

$$
\begin{aligned}
d(S w, z)= & d(S w, T z) \\
\leq & {[\max \{d(A w, B z), d(A w, S w),} \\
& d(B z, T z), d(S w, B z), d(A w, T z)\}]^{\lambda} \\
= & {[\max \{d(z, z), d(z, S w), d(T z, T z), d(S w, z), d(z, z)\}]^{\lambda} . }
\end{aligned}
$$

This implies that $S w=z$. Since $S$ and $A$ are compatible on $X, S w=A w=z$, by Proposition 2.8, we have $A S w=S A w$ and hence $A z=A S w=S A w=S z$. That is, $z=A z=S z=B z=T z$. Therefore, $z$ is common fixed point of $S, T, A$ and $B$.

Similarly, we can complete the proof when $T$ is continuous. 
Finally, suppose that $z$ and $w(z \neq w)$ are two common fixed points of $S, T, A$ and $B$. Consider

$$
\begin{aligned}
d(z, w)= & d(S z, T w) \\
\leq & {[\max \{d(A z, B w), d(A z, S z),} \\
& d(B w, T w), d(S z, B w), d(A z, T w)\}]^{\lambda} \\
= & {[\max \{d(z, w), d(z, z), d(w, w), d(z, w), d(z, w)\}]^{\lambda} } \\
= & d^{\lambda} d(z, w) .
\end{aligned}
$$

This implies that $z=w$. Therefore, $z$ is a unique common fixed point of $S, T, A$ and $B$. This completes the proofs.

Next we give the following theorem for compatible mappings of type $(A)$.

Theorem 3.3. Let $S, T, A$ and $B$ be mappings of a complete multiplicative metric space $(X, d)$ into itself satisfying $(C 1)-(C 3)$.

Assume that the pairs $(A, S)$ and $(B, T)$ are compatible of type $(A)$. Then $S, T, A$ and $B$ have a unique common fixed point.

Proof. Suppose that $A$ is continuous. Since $(A, S)$ are compatible of type $(A)$, by Proposition 2.2, the pair $(A, S)$ is compatible, so result easily follows from Theorem 3.2.

Similarly if $B$ is continuous and $(B, T)$ is compatible of type $(A)$, then $(B, T)$ is compatible so result easily follows from Theorem 3.2.

Similarly, we can complete the proof when $S$ or $T$ is continuous. This completes the proofs.

Also we give the following theorem for compatible mappings of type $(B)$.

Theorem 3.4. Let $S, T, A$ and $B$ be mappings of a complete multiplicative metric space $(X, d)$ into itself satisfying $(C 1)-(C 3)$.

Assume that the pairs $(A, S)$ and $(B, T)$ are compatible of type $(B)$. Then $S, T, A$ and $B$ have a unique common fixed point.

Proof. From the proof of Theorem 3.2, $\left\{y_{n}\right\}$ is multiplicative Cauchy sequence in $X$. Consequently, the subsequences $\left\{S x_{2 n}\right\},\left\{A x_{2 n}\right\},\left\{T x_{2 n+1}\right\}$ and $\left\{B x_{2 n+1}\right\}$ of $\left\{y_{n}\right\}$ converge to $z$.

Suppose that $S$ is continuous. Then $S S x_{2 n}, S A x_{2 n}$ converge to $S z$ as $n \rightarrow$ $\infty$. Since the pair $(A, S)$ is compatible of type $(B)$, it follows from Proposition 2.11 that $A A x_{2 n}$ converges to $S z$ as $n \rightarrow \infty$. 
Consider

$$
\begin{aligned}
d\left(S A x_{2 n}, T x_{2 n+1}\right) \leq[ & \max \left\{d\left(A A x_{2 n}, B x_{2 n+1}\right), d\left(A A x_{2 n}, S A x_{2 n}\right),\right. \\
& d\left(B x_{2 n+1}, T x_{2 n+1}\right), d\left(S A x_{2 n}, B x_{2 n+1}\right) \\
& \left.\left.d\left(\operatorname{Aax}_{2 n}, T x_{2 n+1}\right)\right\}\right]^{\lambda} .
\end{aligned}
$$

Letting $n \rightarrow \infty$, we get

$$
d(S z, z) \leq[\max \{d(S z, z), d(S z, S z), d(z, z), d(S z, z), d(S z, z)\}]^{\lambda} .
$$

This implies that $S z=z$. Since $S X \subset B X$ there exists a point $u \in X$ such that $z=S z=B u$. Consider

$$
\begin{aligned}
& d\left(S A x_{2 n}, T u\right) \leq\left[\operatorname { m a x } \left\{d\left(A A x_{2 n}, B u\right), d\left(A A x_{2 n}, S A x_{2 n}\right),\right.\right. \\
&\left.\left.d(B u, T u), d\left(S A x_{2 n}, B u\right), d\left(A A x_{2 n}, T u\right)\right\}\right]^{\lambda} .
\end{aligned}
$$

Letting $n \rightarrow \infty$, we get

$$
d(S z, T u) \leq d^{\lambda}(S z, T u)
$$

This implies that $T u=S z(z=T u)$. Since the pair $(B, T)$ is compatible of type $(B)$ and $B u=z=T u$. By Proposition 2.10 we have $T B u=B T u$ and so $B z=B T u=T B u=T z$. Consider

$$
\begin{aligned}
d\left(S x_{2 n}, T z\right) \leq\left[\operatorname { m a x } \left\{d\left(A x_{2 n}, B z\right), d\left(A x_{2 n}, S x_{2 n}\right),\right.\right. \\
\\
\left.\left.d(B z, T z), d\left(S x_{2 n}, B z\right), d\left(A x_{2 n}, T z\right)\right\}\right]^{\lambda} .
\end{aligned}
$$

Letting $n \rightarrow \infty$, we get

$$
d(z, T z) \leq d^{\lambda}(z, T z)
$$

which implies that $T z=z$. Since $T X \subset A X$, there exists a point $v \in X$ such that $z=T z=A v$. Consider

$$
\begin{aligned}
d(S v, T z) \leq[\max \{d(A v, B z), d(A v, S v), & \\
& d(B z, T z), d(S v, B z), d(A v, T z)\}]^{\lambda},
\end{aligned}
$$

which implies that

$$
d(S v, z) \leq d^{\lambda}(S v, z)
$$

This implies $S v=z$. Since the pair $(A, S)$ is compatible of type $(B)$ and $S v=z=A v$, it follows from Proposition 2.10 that $S z=S A v=A S v=A z$. Therefore, $A z=B z=S z=T z=z$ and hence $z$ is common fixed point of $S, T, A$ and $B$. 
Now suppose that $A$ is continuous. Then $A A x_{2 n}$ and $A S x_{2 n}$ converge to $A z$ as $n \rightarrow \infty$. Since $(A, S)$ is compatible of type $(B)$, it follows from Proposition 2.11 that $S S x_{2 n}$ converges to $A z$ as $n \rightarrow \infty$. Consider

$$
\begin{aligned}
d\left(S S x_{2 n}, T x_{2 n+1}\right) \leq[ & \max \left\{d\left(A S x_{2 n}, B x_{2 n+1}\right), d\left(A S x_{2 n}, S S x_{2 n}\right),\right. \\
& d\left(B x_{2 n+1}, T x_{2 n+1}\right), d\left(S S x_{2 n}, B x_{2 n+1}\right) \\
& \left.\left.d\left(A S x_{2 n}, T x_{2 n+1}\right)\right\}\right]^{\lambda}
\end{aligned}
$$

Letting $n \rightarrow \infty$, we get

$$
d(A z, z) \leq d^{\lambda}(A z, z)
$$

This implies $A z=z$. Consider

$$
\begin{aligned}
d\left(S z, T x_{2 n+1}\right) \leq[ & \max \left\{d\left(A z, B x_{2 n+1}\right), d(A z, S z),\right. \\
& d\left(B x_{2 n+1}, T x_{2 n+1}\right), d\left(S z, B x_{2 n+1}\right), \\
& \left.\left.d\left(A z, T x_{2 n+1}\right)\right\}\right]^{\lambda} .
\end{aligned}
$$

Letting $n \rightarrow \infty$, we get

$$
d(S z, z) \leq d^{\lambda}(S z, z)
$$

This implies $S z=z$. Since $S X \subset B X$, there exists a point $w \in X$ such that $z=S z=B w$. Consider

$$
\begin{aligned}
d(z, T w)= & d(S z, T u) \\
\leq & {[\max \{d(A z, B w), d(A z, S z),} \\
& d(B w, T w), d(S z, B w), d(A z, T w)\}]^{\lambda} \\
= & {[\max \{d(z, z), d(z, z), d(z, T w), d(z, z), d(z, T w)\}]^{\lambda} . }
\end{aligned}
$$

This implies that $z=T w$. Since $(B, T)$ is compatible of type $(B)$ and $B w=$ $z=T w$, from Proposition 2.10, TBw $=B T w$ and so $B z=B T w=T B w=T z$. Consider

$$
\begin{aligned}
d(S z, T z) & \leq[\max \{d(z, T z), d(z, z), d(T z, T z), d(z, T z), d(z, T z)\}]^{\lambda} \\
& =d^{\lambda}(z, T z) .
\end{aligned}
$$

This implies that $z=T z$. Therefore, $z$ is common fixed point of $S, T, A$ and $B$.

Similarly, we can complete the proof when $B$ or $T$ is continuous.

Finally, if $z$ and $w(z \neq w)$ are two common fixed points, then we have

$$
\begin{aligned}
d(z, w)= & d(S z, T w) \\
\leq & {[\max \{d(A z, B w), d(A z, S z),} \\
& \quad d(B w, T w), d(S z, B w), d(A z, T w)\}]^{\lambda} \\
= & d^{\lambda}(z, w) .
\end{aligned}
$$


This implies that $z=w$. Therefore, $z$ is a unique common fixed point of $S, T, A$ and $B$. This completes the proofs.

Now we give the following theorem for compatible mappings of type $(C)$.

Theorem 3.5. Let $S, T, A$ and $B$ be mappings of a multiplicative metric space $(X, d)$ into itself satisfying $(C 1)-(C 3)$.

Assume that the pair $(A, S)$ and $(B, T)$ are compatible of type $(C)$. Then $S, T, A$ and $B$ have a unique common fixed point.

Proof. From the proof of Theorem 3.2, $\left\{y_{n}\right\}$ is multiplicative Cauchy sequence in $X$. Consequently, the subsequences $\left\{S x_{2 n}\right\},\left\{A x_{2 n}\right\},\left\{T x_{2 n+1}\right\}$ and $\left\{B x_{2 n+1}\right\}$ of $\left\{y_{n}\right\}$ also converge to $z$.

Suppose that $S$ is continuous. Then $S S x_{2 n}, S A x_{2 n}$ converge to $S z$ as $n \rightarrow$ $\infty$. Since the pair $(A, S)$ is compatible of type $(C)$, it follows from Remark 2.13 that $A A x_{2 n}$ converges to $S z$ as $n \rightarrow \infty$.

We claim that $S z=z$. Consider

$$
\begin{aligned}
d\left(S A x_{2 n}, T x_{2 n+1}\right) \leq[ & \max \left\{d\left(A A x_{2 n}, B x_{2 n+1}\right), d\left(A A x_{2 n}, S A x_{2 n}\right),\right. \\
& d\left(B x_{2 n+1}, T x_{2 n+1}\right), d\left(S A x_{2 n}, B x_{2 n+1}\right), \\
& \left.\left.d\left(A A x_{2 n}, T x_{2 n+1}\right)\right\}\right]^{\lambda} .
\end{aligned}
$$

Letting $n \rightarrow \infty$, we get

$$
\begin{aligned}
d(S z, z) & \leq[\max \{d(A z, z), d(S z, S z), d(z, z), d(S z, z), d(S z, z)\}]^{\lambda} \\
& =d^{\lambda}(S z, z) .
\end{aligned}
$$

This implies that $S z=z$. Since $S X \subset B X$, there exists a point $u \in X$ such that $z=S z=B u$. Consider

$$
\begin{aligned}
d\left(S A x_{2 n}, T w\right) \leq & {\left[\operatorname { m a x } \left\{d\left(A A x_{2 n}, B w\right), d\left(A A x_{2 n}, S A x_{2 n}\right),\right.\right.} \\
& \left.\left.d(B u, T u), d\left(S A x_{2 n}, B u\right), d\left(A A x_{2 n}, T u\right)\right\}\right]^{\lambda} .
\end{aligned}
$$

Letting $n \rightarrow \infty$, we get

$$
\begin{aligned}
d(S z, T u) \leq & {[\max \{d(S z, S z), d(S z, S z),} \\
& d(S z, T u), d(B u, B u), d(S z, T u)\}]^{\lambda} \\
= & d^{\lambda}(S z, T u) .
\end{aligned}
$$


This implies that $S z=T u(z=T u)$. Since the pair $(B, T)$ is compatible of type $(C)$ and $B u=z=T u$, by Remark 2.12, we get $T B u=B T u$ and so $B z=B T u=T B u=T z$. Consider

$$
\begin{aligned}
d\left(S x_{2 n}, T z\right) \leq\left[\operatorname { m a x } \left\{d\left(A x_{2 n}, B z\right), d\left(A x_{2 n}, S x_{2 n}\right),\right.\right. \\
\\
\left.\left.d(B z, T z), d\left(S x_{2 n}, B z\right), d\left(A x_{2 n}, T z\right)\right\}\right]^{\lambda} .
\end{aligned}
$$

Letting $n \rightarrow \infty$, we have

$$
\begin{aligned}
d(z, T z) & \leq[\max \{d(z, T z), d(z, z), 1, d(z, T z), d(z, T z)\}]^{\lambda} \\
& =d^{\lambda}(z, T z) .
\end{aligned}
$$

This implies that $T z=z$. Since $T X \subset A X$, there exists a point $v \in X$ such that $z=T z=A v$. Consider

$$
\begin{aligned}
d(S v, z)= & d(S v, T z) \\
\leq & {[\max \{d(A v, B z), d(A v, S v)} \\
& d(B z, T z), d(S v, B z), d(A v, T z)\}]^{\lambda} \\
\leq & {[\max \{d(z, z), d(S v, z), d(z, z), d(S v, z), d(z, z)\}]^{\lambda} } \\
= & d^{\lambda}(S v, z) .
\end{aligned}
$$

This implies that $z=S v$. Since the pair $(A, S)$ is compatible of type $(C)$ and $S v=z=A v$, by Remark 2.12, $A S v=S A v$ we have $S z=S A v=A S v=A z$. Therefore $B z=A z=T z=S z=z$ and hence $z$ is common fixed point of $S, T, A$ and $B$.

Suppose that $A$ is continuous. Then $A A x_{2 n}$ and $A S x_{2 n}$ converge to $A z$ as $n \rightarrow \infty$. Since the pair $(A, S)$ is compatible of type $(C)$, it follows from Remark 2.13 that $S S x_{2 n}$ converges to $A z$ as $n \rightarrow \infty$.

Also we have

$$
\begin{aligned}
d\left(S S x_{2 n}, T x_{2 n+1}\right) \leq[ & \max \left\{d\left(A S x_{2 n}, B x_{2 n+1}\right), d\left(A S x_{2 n}, S S x_{2 n}\right)\right. \\
& d\left(B x_{2 n+1}, T x_{2 n+1}\right), d\left(S S x_{2 n}, B x_{2 n+1}\right) \\
& \left.\left.d\left(A S x_{2 n}, T x_{2 n+1}\right)\right\}\right]^{\lambda}
\end{aligned}
$$

Letting $n \rightarrow \infty$, we get

$$
\begin{aligned}
d(A z, z) & \leq[\max \{d(A z, z), d(A z, A v), d(z, z), d(A z, z), d(A z, z)\}]^{\lambda} \\
& =d^{\lambda}(A z, z) .
\end{aligned}
$$


This implies that $A z=z$. Consider

$$
\begin{aligned}
d\left(S z, T x_{2 n+1}\right) \leq[ & \max \left\{d\left(A z, B x_{2 n+1}\right), d(A z, S z),\right. \\
& d\left(B x_{2 n+1}, T x_{2 n+1}\right), d\left(S z, B x_{2 n+1}\right), \\
& \left.\left.d\left(A z, T x_{2 n+1}\right)\right\}\right]^{\lambda} .
\end{aligned}
$$

Letting $n \rightarrow \infty$, we get

$$
d(S z, z) \leq d^{\lambda}(S z, z)
$$

This implies $S z=z$. Since $S X \subset B X$, there exists a point $w \in X$ such that $z=S z=B w$. Again, we have

$$
\begin{aligned}
d(z, T w)= & d(S z, T w) \\
\leq & {[\max \{d(A z, B w), d(A z, S z),} \\
& \quad d(B w, T w), d(S z, B w), d(A z, T w)\}]^{\lambda} \\
= & d^{\lambda}(z, T w) .
\end{aligned}
$$

This implies that $T w=z$. Since $(B, T)$ is compatible of type $(C)$ and $B w=z=$ $T w$, by Remark 2.12 we have $T B w=B T w$ and so $B z=B T w=T B w=T z$. Also consider

$$
d(z, T z)=d(S z, T z) \leq d^{\lambda}(z, T z) .
$$

Thus implies that $T z=z$. Hence $T z=B z=S z=A z=z$. Therefore, $z$ is the common fixed point of $S, T, A$ and $B$.

Similarly, we can complete the proof when $B$ or $T$ is continuous.

Uniqueness follows easily. This completes the proofs.

Finally we give the following theorem for compatible mappings of type $(P)$.

Theorem 3.6. Let $S, T, A$ and $B$ be mappings of a complete multiplicative metric space $(X, d)$ into itself satisfying $(C 1)-(C 3)$.

Assume that the pairs $(A, S)$ and $(B, T)$ are compatible of type $(P)$. Then $S, T, A$ and $B$ have a unique common fixed point.

Proof. From the proof of Theorem 3.2, $\left\{y_{n}\right\}$ is multiplicative Cauchy sequence in $X$. Consequently, the subsequences $\left\{S x_{2 n}\right\},\left\{A x_{2 n}\right\},\left\{T x_{2 n+1}\right\}$ and $\left\{B x_{2 n+1}\right\}$ of $\left\{y_{n}\right\}$ converge to $z$ as $n \rightarrow \infty$.

Suppose that $S$ is continuous. Then $S S x_{2 n}, S A x_{2 n}$ converge to $S z$ as $n \rightarrow$ $\infty$. Since $(A, S)$ is compatible of type $(P)$, it follows from Remark 2.13 that $A A x_{2 n}$ converges to $S z$ as $n \rightarrow \infty$. 
We claim $S z=z$. Consider

$$
\begin{aligned}
d\left(S A x_{2 n}, T x_{2 n+1}\right) \leq[ & \max \left\{d\left(A A x_{2 n}, B x_{2 n+1}\right), d\left(A A x_{2 n}, S A x_{2 n} x\right),\right. \\
& d\left(B x_{2 n+1}, T x_{2 n+1}\right), d\left(S A x_{2 n} x, B x_{2 n+1}\right), \\
& \left.\left.d\left(A A x_{2 n}, T x_{2 n+1}\right)\right\}\right]^{\lambda} .
\end{aligned}
$$

Letting $n \rightarrow \infty$, we have

$$
\begin{aligned}
d(S z, z) & \leq[\max \{d(S z, z), d(S z, S z), d(z, z), d(S z, z), d(S z, z)\}]^{\lambda} \\
& =d^{\lambda}(S z, z) .
\end{aligned}
$$

This implies that $S z=z$. Since $S X \subset B X$, so there exists a point $u \in X$ such that $z=S z=B u$.

Now we claim that $T u=z$. Consider

$$
\begin{aligned}
d\left(S x_{2 n}, T u\right) \leq\left[\operatorname { m a x } \left\{d\left(A x_{2 n}, B u\right), d\left(A x_{2 n}, S x_{2 n}\right),\right.\right. & \\
& \left.\left.d(B u, T u), d\left(S x_{2 n}, B u\right), d\left(A x_{2 n}, T u\right)\right\}\right]^{\lambda} .
\end{aligned}
$$

Letting $n \rightarrow \infty$, we get

$$
\begin{aligned}
d(z, T u) & \leq[\max \{d(z, z), d(z, z), d(z, T u), d(z, z), d(z, T u)\}]^{\lambda} \\
& =d^{\lambda}(z, T u) .
\end{aligned}
$$

This implies that $z=T u$. Therefore, $B u=T u=z$. Since $(B, T)$ is compatible of type $(P)$, by Remark 2.12, we have $T T u=B B u$, which implies that $d(B z, T z)=1$. Hence $T z=B z$.

Now we claim that $T z=z$. Consider

$$
\begin{aligned}
& d\left(S x_{2 n}, T z\right) \leq\left[\operatorname { m a x } \left\{d\left(A x_{2 n}, B z\right), d\left(A x_{2 n}, S x_{2 n}\right),\right.\right. \\
&\left.\left.d(B z, T z), d\left(S x_{2 n}, B z\right), d\left(A x_{2 n}, T z\right)\right\}\right]^{\lambda} .
\end{aligned}
$$

Letting $n \rightarrow \infty$, we have

$$
d(z, T z) \leq d^{\lambda}(z, T z)
$$

which implies that $z=T z$. Therefore, $B z=T z=z$. Since $T X \subset A X$, so there exists a point $v \in X$ such that $z=T z=A v$.

Now to claim that $S v=z$. Consider

$$
\begin{aligned}
d(S v, z)= & d(S v, T z) \\
\leq & {[\max \{d(A v, B z), d(A v, S v),} \\
& \quad d(B z, T z), d(S v, B z), d(A v, T z)\}]^{\lambda} \\
= & d^{\lambda}(S v, z) .
\end{aligned}
$$


This implies that $z=S v$. Therefore $z=S v=A v$. Since $(A, S)$ is compatible of type $(P)$, by Remark 2.12, we have $S S v=A A v$, which implies that $d(S z, A z)=$ 1. Hence $S z=A z$. Since $A z=B z=S z=T z=z, z$ is common fixed point of $S, T, A$ and $B$.

Similarly, we can complete the proof when $A$ or $B$ or $T$ is continuous.

The uniqueness follows easily. This completes the proofs.

Now we give an example in support of our main theorems.

Example 3.7. Let $X=[1, \infty)$ with usual multiplicative metric $d(x, y)=$ $\left|\frac{x}{y}\right|$. Consider the following self-mappings $S x=x, T x=x^{2}, B x=2 x^{4}-1$ and $A x=2 x^{2}-1$ for all $x \geq 1$.

$S X=T X=B X=A X=X, S X \subset B X, T X \subset A X ;$

$S, T, A$ and $B$ are all continuous mappings;

(iii) the pairs $(A, S)$ and $(B, T)$ are compatible, and they are compatible mappings of type $(A)$, of type $(B)$, of type $(C)$ and of type $(P)$.

Consider $x_{n}=1+\frac{1}{n}$ for $n \geq 1$. Then $x_{n} \rightarrow 1$ as $n \rightarrow \infty$. Now we have

$$
\lim _{n \rightarrow \infty} A x_{n}=\lim _{n \rightarrow \infty} S x_{n}=\lim _{n \rightarrow \infty} B x_{n}=\lim _{n \rightarrow \infty} T x_{n}=1=t \in X
$$

as $n \rightarrow \infty$. Also we have

$$
\begin{array}{cc}
\lim _{n \rightarrow \infty} d\left(A S x_{n}, S A x_{n}\right)=1, & \lim _{n \rightarrow \infty} d\left(B T x_{n}, T B x_{n}\right)=1, \\
\lim _{n \rightarrow \infty} d\left(A S x_{n}, S S x_{n}\right)=1, & \lim _{n \rightarrow \infty} d\left(S A x_{n}, A A x_{n}\right)=1, \\
\lim _{n \rightarrow \infty} d\left(B T x_{n}, T T x_{n}\right)=1, & \lim _{n \rightarrow \infty} d\left(T B x_{n}, B B x_{n}\right)=1 .
\end{array}
$$

(iv) For $\lambda=\frac{1}{3}$,

$$
\begin{aligned}
d(S x, T y) \leq & {[\max \{d(A x, B y), d(A x, S x),} \\
& d(B y, T y), d(S x, B y), d(A x, T y)\}]^{\lambda}
\end{aligned}
$$

is satisfied for all $x, y \in X$. Therefore, all conditions of main theorems are satisfied, and 1 is a unique common fixed point of $S, T, A$ and $B$.

\section{References}


[1] A.E. Bashirov, E.M. Kurplnara, A. Ozyapici, Multiplicative calculus and its applications, J. Math. Anal. Appl., 337 (2008), 36-48. doi: 10.1016/j.jmaa.2007.03.081

[2] X. He, M. Song, D. Chen, Common fixed points for weak commutative mappings on a multiplicative metric space, Fixed Point Theory Appl., 48 (2014), 9 pages. doi: 10.1186/1687-1812-2014-48

[3] F. Gu, L.M. Cui, Y.H. Wu, Some fixed point theorems for new contractive type mappings, J. Qiqihar Univ., 19 (2013), 85-89.

[4] M. Özavsar, A.C. Cevikel, Fixed point of multiplicative contraction mappings on multiplicative metric space, arXiv:1205.5131v1 [matn.GM], 2012.

[5] M. Sarwar, R. Badshah-e, Some unique fixed point theorems in multiplicative metric space, arXiv:1410.3384v2 [math.GM], 2014. 